\title{
Molecular Dynamics Simulations of Heavy Ion Induced Defects in SiC Schottky Diodes
}

\author{
Javanainen, Arto
}

2018-09

Javanainen , A , Muinos , H V , Nordlund, K, Djurabekova , F , Galloway , K F , Turowski , M \& Schrimpf , R D 2018 , ' Molecular Dynamics Simulations of Heavy Ion Induced Defects in SiC Schottky Diodes ', IEEE transactions on device and materials reliability. , vol. 18 , no. 3 , pp. 481-483 . https://doi.org/10.1109/TDMR.2018.2842253

http://hdl.handle.net/10138/308631

https://doi.org/10.1109/TDMR.2018.2842253

acceptedVersion

Downloaded from Helda, University of Helsinki institutional repository.

This is an electronic reprint of the original article.

This reprint may differ from the original in pagination and typographic detail.

Please cite the original version. 


\title{
Molecular dynamics simulations of heavy ion induced defects in SiC Schottky diodes
}

\author{
Arto Javanainen, Member, IEEE, Henrique Vazquez Muinos, Kai Nordlund, \\ Flyura Djurabekova Kenneth F. Galloway, Life Fellow, IEEE, \\ Marek Turowski, Senior Member, IEEE, Ronald D. Schrimpf, Fellow, IEEE
}

\begin{abstract}
Heavy ion irradiation increases the leakage current in reverse-biased $\mathrm{SiC}$ Schottky diodes. This work demonstrates, via molecular dynamics simulations, that a combination of bias and ion-deposited energy is required to produce the degradation.
\end{abstract}

Index Terms - Ion radiation effects, Modeling, Power semiconductor devices, Schottky diodes, Silicon carbide

\section{INTRODUCTION}

$\mathrm{S}_{\mathrm{t}}$ ILICON CARBIDE ( $\mathrm{SiC}$ ) power devices are of great interest for their possible use in power applications in spaceborne electronics. However, SiC power devices (MOSFETs and diodes) are sensitive to heavy-ion particle radiation such as that found in space. Javanainen et al. [1] previously discussed the leakage current in SiC Schottky diodes resulting from heavyion exposure. This paper examines the physical processes that result in that leakage current.

SiC Schottky diodes may exhibit Single Event Burnout (SEB) similar to that observed in silicon-based power devices; at bias voltages below the SEB threshold, however, SiC Schottky diodes also exhibit ion-induced increases of reverse leakage current, as illustrated in Figure 1. The leakage current may begin to increase during irradiation at operating biases significantly lower than the rated breakdown voltage [1]-[8]. For the $650 \mathrm{~V}$ devices in this work, semi-permanent increases in ion-induced leakage occur at a bias as low as $150 \mathrm{~V}$. Device simulations have been used to attribute this to a highly localized temperature rise that goes above the phase transition temperature of $\mathrm{SiC}$ [4], [5]. Even though the temperature recovers after the ion strike, physical modifications of the crystal occur, as evidenced by the changes in the current-voltage characteristics. Simulated temperature effects due to ion and bias also have been reported by Abbate et al. [7], [8] and by Witulski et al. [6].

In order to study the permanent defects generated by the heavy-ion and bias-induced Joule heating, we use molecular dynamics (MD) to simulate the effect of ion impact on the $\mathrm{SiC}$ crystal structure. The ion energy deposition in these simulations

This work was supported by the European Space Agency (ESA/ESTEC Contract 4000111630/14/NL/PA), and the Academy of Finland under the Finnish Centre of Excellence Programme 2012-2017 (Project No 2513553, Nuclear and Accelerator Based Physics). It was also funded in part, by an Early State Innovations grant (NNX17AD09G) from NASA's Space Technology Research Grants Program, and the MATRENA doctoral programme and the Academy of Finland (Grant No 1269 696).

A. Javanainen is with the University of Jyvaskyla, Department of Physics, P.O. Box 35, FI-40014, University of Jyvaskyla, Finland. (email: arto.javanainen@jyu.fi)

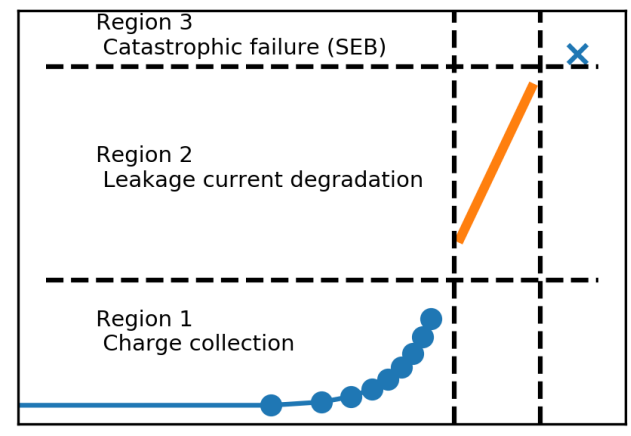

Bias voltage

Figure 1. Three response regions observed in $\mathrm{SiC}$ diodes exposed to heavy-ion irradiation. After Kuboyama et al. [2].

is defined by the average Linear Energy Transfer (LET) (also known as electronic stopping force, $\mathrm{S}_{\mathrm{e}}$ ) value for $1217-\mathrm{MeV} \mathrm{Xe}$ ions, that is $62.4 \mathrm{MeV} /\left(\mathrm{mg} / \mathrm{cm}^{2}\right)$. This corresponds to experimental values used earlier [1], [4], [5].

Because MD simulations cannot include the electric field or current transport, technology computer-aided design (TCAD) simulations are used to obtain the temporal and spatial temperature and carrier distributions in biased devices. These data are used as input for MD simulations that describe the response of the $\mathrm{SiC}$ lattice structure to the energy dissipation due to the Joule heating.

\section{EXPERIMENTAL AND TCAD RESULTS}

The experimental results used to inform the simulations presented in this paper have been published previously [1], [4], [5].

The SiC Schottky diodes exhibit strong dependence on the ion species (or LET) and reverse bias applied during the irradiation. The current work concentrates only on Xe-ions.

TCAD simulations have indicated that the synergetic effects between the heavy-ion induced ionization and the applied bias lead to $\mathrm{SiC}$ lattice temperatures that exceed the phase transition point of the material. This is illustrated in Figure 2, where the

A. Javanainen is also with the Electrical Engineering and Computer Science Department, Vanderbilt University, Nashville, TN 37235 USA. (email: arto.javanainen@vanderbilt.edu)

H. Vazquez Muinos, K. Nordlund, and F. Djurabekova are with Helsinki Institute of Physics and Department of Physics, University of Helsinki, P.O. Box 43, FI-00014, Finland

K. Galloway and R. Schrimpf are with the Electrical Engineering and Computer Science Department, Vanderbilt University, Nashville, TN 37235 USA. (email: \{ken.galloway, ron.schrimpf\}@vanderbilt.edu)

M. Turowski is with Silvaco Inc., 2811 Mission College Blvd, 6th Floor, Santa Clara, CA 95054, USA, (email: marek.turowski@silvaco.com) 
TCAD-simulated time evolution of the lattice temperature is presented for four different bias conditions.

However, TCAD simulations are not capable of giving information on material changes that may occur at these high temperatures. Hence, for ion-induced damage formation we used molecular dynamics simulations that are discussed below.

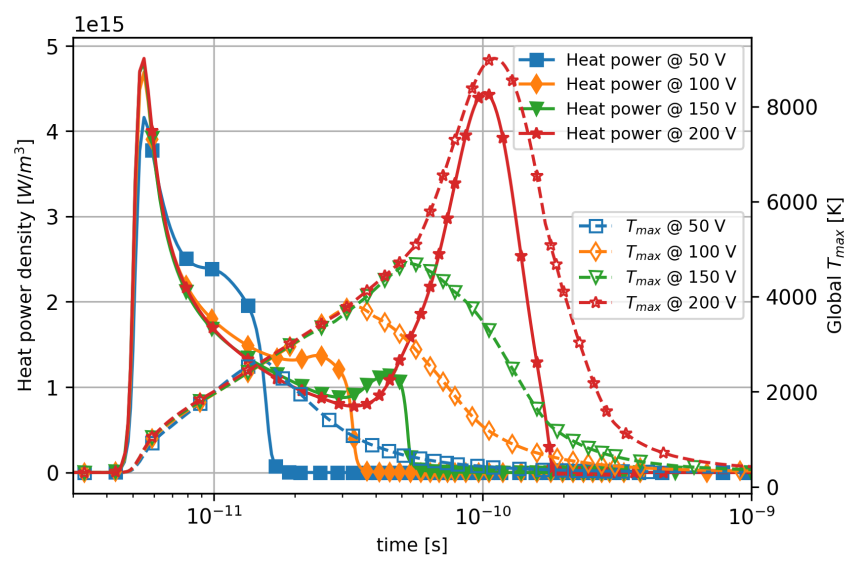

Figure 2. TCAD simulated temporal evolution of the heat power densities (solid) and the maximum lattice temperature (dashed) in SiC Schottky diode structure after Xe ion strike. The average LET value of $62.4 \mathrm{MeV} /\left(\mathrm{mg} / \mathrm{cm}^{2}\right)$ is used for estimating the energy deposition by the ion. Different curves represent different reverse bias voltages applied on the Schottky diode.

\section{MD SIMULATIONS}

The molecular dynamics (MD) code PARCAS [9] is used to simulate the effects of heavy-ion impacts on different model damage states in $4 \mathrm{H}-\mathrm{SiC}$ at room temperature. The Erhart-Albe $\mathrm{SiC}$ potential [10] is used to describe the $\mathrm{Si}-\mathrm{C}$ interactions. This potential predicts a melting point between $3500-4000 \mathrm{~K}$ [11], compared to the experimental value of $3000 \mathrm{~K}$ [12]. In order to simulate the effect of energetic heavy ions in classical MD simulations (where electrons are not present explicitly), the heat added to the lattice due to the electronic excitations is estimated utilizing the inelastic thermal spike model [13]. In this model, two heat equations for electronic and atomic lattice subsystems, coupled through the electron-phonon coupling constant, are solved concurrently.

The energy deposited initially by the heavy-ion into the electronic subsystem is estimated from the electronic stopping power function of the ion in $\mathrm{SiC}$ as given by SRIM [14]. Solving these equations, one can estimate the evolution of the temperature in the lattice as a function of time and radial distance from the ion trajectory.

In order to see the effect of bias and find the correlation with the experimental results, we simulate the impact of a $1217-\mathrm{MeV}$ Xe ion with an LET of $62.4 \mathrm{MeV} /\left(\mathrm{mg} / \mathrm{cm}^{2}\right)$ in the SiC lattice with and without applied bias. In the heavy-ion experiments the unbiased case means that all the device pins are grounded.

$$
\begin{aligned}
& C_{e} \frac{\partial T_{e}}{\partial t}=\nabla\left[K_{e}\left(\mathrm{~T}_{\mathrm{e}}\right) \cdot \nabla \mathrm{T}_{\mathrm{e}}\right]-g\left(T_{e}-T_{l}\right)+A(r, t) \\
& C_{l} \frac{\partial T_{l}}{\partial t}=\nabla\left[K_{l}\left(\mathrm{~T}_{1}\right) \cdot \nabla \mathrm{T}_{l}\right]+g\left(T_{e}-T_{l}\right)
\end{aligned}
$$

For the case without bias, we calculate the evolution of the electronic and lattice subsystems according to the thermal spike model (see eqns. 1 and 2), and use the same electronic parameters as in previous work [15]. The parameters employed are: electronic specific heat capacity $C_{e}=1 \mathrm{Jcm}^{-3} \mathrm{~K}^{-1}$ [16], electronic heat conductivity $K_{e}=C_{e} D_{e}$ with diffusivity $D_{e}=$ $2 \mathrm{~cm}^{2} \mathrm{~s}^{-1}$ [16], and electron-phonon coupling constant $g=$ $K_{e} / \lambda$ [16]. In the latter, we estimate the electron-phonon mean free path to be $\lambda=5.6 \mathrm{~nm}$ as in [15]. Furthermore, we obtain the lattice parameters used in the calculation from [17], [18]. We give the initial energy deposition to the electron subsystem $A(r, t)$ according to the distribution from Waligorski et al. [19]; this distribution was obtained as a fit to Monte Carlo simulations of ion-induced electronic cascades. We calculate analytically the temperature evolution in the electron and lattice coupled subsystems until $100 \mathrm{fs}$, when the electrons have transmitted most of their energy to the lattice. We extract the lattice temperature profile at this moment and deposit the corresponding kinetic energy to the atoms in the MD cell. The MD simulations are used to obtain the structure of the track created by the passing ion after the first $100 \mathrm{ps}$ when the energy has already dissipated into the bulk and the atoms in the core of the track have cooled down. The dimensions of the simulation cell used were $30 \times 30 \times 5 \mathrm{~nm}^{3}$.

For the case when bias is applied, we use the results from previous TCAD work [5], where the evolution of the heat power density with time was calculated for the same ion as above with $200 \mathrm{~V}$ applied. The evolution of the system is simulated with $\mathrm{MD}$, while adding the time-dependent heat power distributions from the TCAD calculations. The dimensions of the simulation cell used were $92 \times 105 \times 5 \mathrm{~nm}^{3}$.

The TCAD simulations show large spatial spread (around 40 $\mathrm{nm}$ ) of the Joule heating, however due to limited computational resources we use a spatial Gaussian distribution of $23 \mathrm{~nm}$ FWHM. Therefore, these results correspond to a lower bound estimate of the damage produced by the irradiation with bias. The displacements of the atoms and the appearance of structural deformations due to the Joule heating are obtained from MD.

The results are indicative, as the phase transition point of the MD potential used here might not reproduce correctly the phase transition point of the $\mathrm{SiC}$, underestimating the appearance of defects in the material.

\section{RESULTS AND DISCUSSION}

Figure 3 shows the radial distributions of the maximum temperature after Xe-ion strikes at different biases, obtained from the MD simulations. Without an applied bias, the lattice temperature rises slightly, but remains below the $\mathrm{SiC}$ melting temperature $(3500-4000 \mathrm{~K})[11]$. Also at $50 \mathrm{~V}$ bias, the lattice temperatures obtained from both TCAD and MD simulations are relatively low. However, when higher bias is applied, the simulated lattice temperatures reach much higher values. Combining this with the fact that the thermal runaway temperature within $\mathrm{SiC}$ diode structures has been shown [20] to decrease with increasing reverse bias voltage, the simulation results support the idea that ion strikes at higher bias voltages are more 
capable of generating lattice damage. Figure 4 illustrates the lattice structure after an ion strike with 0 and $200 \mathrm{~V}$ bias.

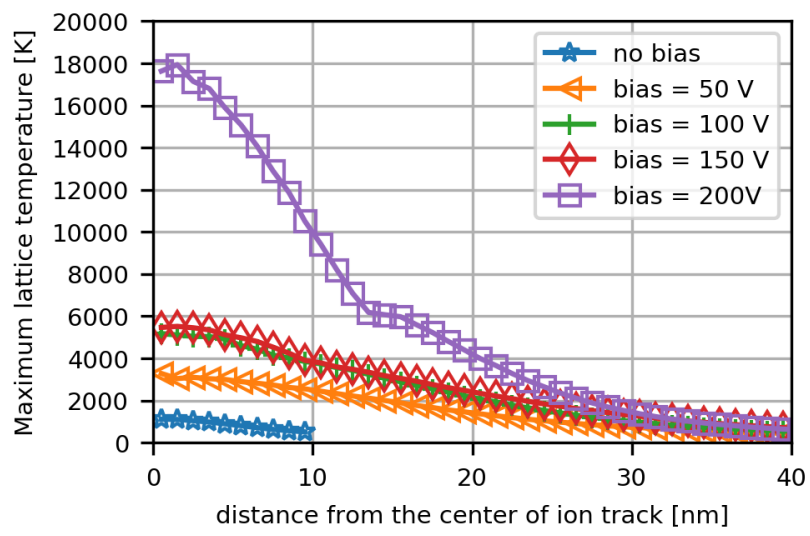

Figure 3. Radial distribution of the maximum temperature within the $4 \mathrm{H}-\mathrm{SiC}$ lattice after the ion strike at different bias configurations.

At biases of $50 \mathrm{~V}$ and below the simulations do not show any permanent damage in the lattice. Figure 5 presents the radius of the ion-induced damage site within the $\mathrm{SiC}$ lattice as a function of applied bias as obtained from the MD simulations. In the experimental results reported earlier [4], the onset for permanent damage induced by Xe-ions was at about $130 \mathrm{~V}$. The difference in the damage onset between simulations $(50 \mathrm{~V})$ and experiments $(130 \mathrm{~V})$ can be explained by the limited size of the volume considered in the simulations. In real devices, the Joule heat can dissipate in much larger volume compared to the simulated structure. Also, the top metal layers, that can affect the heat transport, are not taken into account in the simulations.

All in all, the results agree with previous experimental results that have not shown any heavy-ion induced permanent damage in $\mathrm{SiC}$ at sufficiently low biases [4], or without any external electric fields [21]. The results with the higher applied bias suggest, however, that heavy-ion exposure can result in amorphous regions in the material about $10 \mathrm{~nm}$ in size. In the simulations, the energy deposited by Joule heating is very high, and sufficient to cause a phase transition in the material despite the high thermal conductivity of $\mathrm{SiC}$. Even though the recrystallization rate of $\mathrm{SiC}$ is high, based on previous experience [15], it is unlikely that the material is able to recrystallize back completely to eliminate such a large defect, leaving permanent structural modifications in the material.

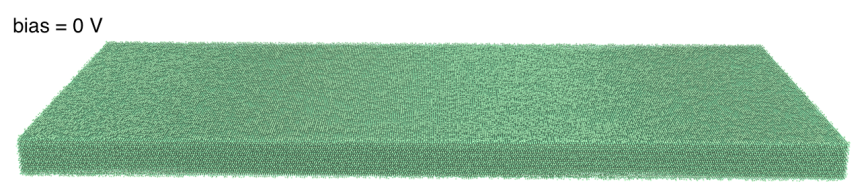

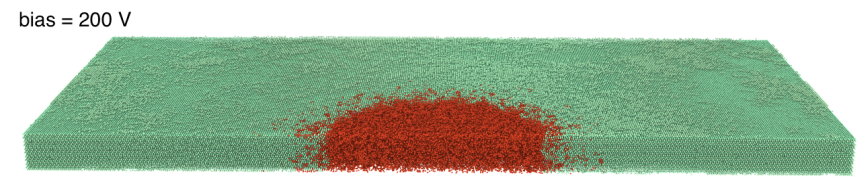

Figure 4. Simulation cell used in the MD simulations showing no damage (top) for $\mathrm{OV}$ bias and damage (bottom) for $200 \mathrm{~V}$ bias. The size is $92 \times 105 \times 5 \mathrm{~nm}^{3}$. Material is $4 \mathrm{H}-\mathrm{SiC}$. The atoms are colored blue or red depending on if their local surrounding is crystalline or amorphous respectively.

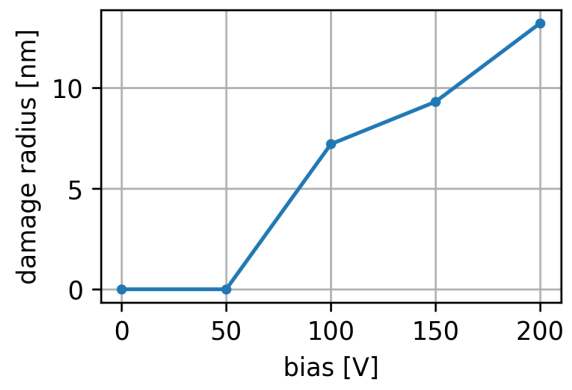

Figure 5. The MD simulated radius of ion-induced damage site in $\mathrm{SiC}$ as a function of applied bias voltage.

These simulations suggest that the Joule heating induced by the interplay of the ionization by the impinging ion, and the applied bias, is capable of producing permanent defects in SiC lattice. However, an ion, impinging on the material with sufficiently low or zero electric field, is not. The nanoscale defects observed in this work could potentially explain the source of the leakage current observed in the experiments under heavy-ion irradiation with applied bias.

\section{CONCLUSIONS}

Molecular dynamics simulations are used to examine heavyion induced material modifications in biased SiC Schottky diodes. The high power dissipation along the ion track in the biased structure results in violent lattice vibrations that can lead to permanent lattice damage if the bias voltage is sufficient.

The MD simulations suggest that there is a threshold voltage for the lattice damage, supporting recent experimental findings.

\section{ACKNOWLEDGMENTS}

The authors would like to thank Jean-Marie Lauenstein at NASA GSFC and Robert Weller, Robert Reed, Art Witulski and Dennis Ball at Vanderbilt University for discussions of heavy-ion radiation effects in SiC Schottky diodes. Some of these results were presented at the 2017 RADECS Conference in Geneva, October 2017.

\section{REFERENCES}

[1] A. Javanainen et al., "Charge Transport Mechanisms in Heavy-Ion Driven Leakage Current in Silicon Carbide Schottky Power Diodes," IEEE Trans. Device Mater. Reliab., vol. 16, no. 2, pp. 208-212, Jun. 2016.

[2] S. Kuboyama, C. Kamezawa, Y. Satoh, T. Hirao, and H. Ohyama, "Single-Event Burnout of Silicon Carbide 
Schottky Barrier Diodes Caused by High Energy Protons," IEEE Trans. Nucl. Sci., vol. 54, no. 6, pp. 2379-2383, Dec. 2007.

[3] S. Kuboyama, C. Kamezawa, N. Ikeda, T. Hirao, and H. Ohyama, "Anomalous Charge Collection in Silicon Carbide Schottky Barrier Diodes and Resulting Permanent Damage and Single-Event Burnout," IEEE Trans. Nucl. Sci., vol. 53, no. 6, pp. 3343-3348, Dec. 2006.

[4] A. Javanainen et al., "Heavy Ion Induced Degradation in SiC Schottky Diodes: Bias and Energy Deposition Dependence," IEEE Trans. Nucl. Sci., vol. 64, no. 1, pp. 415-420, Jan. 2017.

[5] A. Javanainen et al., "Heavy Ion Induced Degradation in SiC Schottky Diodes: Incident Angle and Energy Deposition Dependence," IEEE Trans. Nucl. Sci., vol. 64, no. 8, pp. 2031-2037, 2017.

[6] A. F. F. Witulski et al., "Single-Event Burnout of SiC Junction Barrier Schottky Diode High-Voltage Power Devices," IEEE Trans. Nucl. Sci., vol. 65, no. 1, pp. 256 261, Jan. 2018.

[7] C. Abbate et al., "Thermal damage in SiC Schottky diodes induced by SE heavy ions," Microelectron. Reliab., vol. 54, no. 9-10, pp. 2200-2206, Sep. 2014.

[8] C. Abbate et al., "Analysis of Heavy Ion Irradiation Induced Thermal Damage in SiC Schottky Diodes," IEEE Trans. Nucl. Sci., vol. 62, no. 1, pp. 202-209, Feb. 2015.

[9] K. Nordlund, "PARCAS code." 2006.

[10] P. Erhart and K. Albe, "Analytical potential for atomistic simulations of silicon, carbon, and silicon carbide," Phys. Rev. B, vol. 71, no. 3, p. 35211, Jan. 2005.

[11] S. Z. Chavoshi and X. Luo, "Atomic-scale characterization of occurring phenomena during hot nanometric cutting of single crystal 3C-SiC," RSC Adv., vol. 6, no. 75, pp. 7140971424, Jul. 2016.

[12] W. M. Haynes, Ed., CRC Handbook of Chemistry and Physics. Boca Raton, FL: CRC Press, 2011.

[13] M. Toulemonde, W. Assmann, C. Dufour, A. Meftah, F. Studer, and C. Trautmann, "Experimental Phenomena and Thermal Spike Model Description of Ion Tracks in Amorphisable Inorganic Insulators," Mat. Meddelelser Det K. Danske Vidensk. Selsk., vol. 52, p. 263, 2006.

[14] J. F. Ziegler et al., "SRIM 2013 code." [Online]. Available: http://www.srim.org.

[15] M. Backman et al., "Molecular dynamics simulations of swift heavy ion induced defect recovery in SiC," Comput. Mater. Sci., vol. 67, pp. 261-265, Feb. 2013.

[16] M. Toulemonde, C. Dufour, A. Meftah, and E. Paumier, "Transient thermal processes in heavy ion irradiation of crystalline inorganic insulators," Nucl. Instruments Methods Phys. Res. Sect. B Beam Interact. with Mater. Atoms, vol. 166-167, pp. 903-912, May 2000.

[17] J. Karditsas and M.-J. Baptiste, "Thermal and Structural Properties of Fusion related Materials." [Online]. Available: http://www-ferp.ucsd.edu/LIB/PROPS/PANOS/sic.html.

[18] Y. S. Touloukian, Ed., Thermophysical Properties of High Temperature Solid Materials: Elements. New York: Macmillan Co., 1967.

[19] M. P. R. Waligórski, R. N. Hamm, and R. Katz, "The radial distribution of dose around the path of a heavy ion in liquid water," Int. J. Radiat. Appl. Instrumentation. Part D. Nucl. Tracks Radiat. Meas., vol. 11, no. 6, pp. 309-319, Jan. 1986.

[20] C. Buttay, C. Raynaud, H. Morel, G. Civrac, M.-L. Locatelli, and F. Morel, "Thermal Stability of Silicon Carbide Power Diodes," IEEE Trans. Electron Devices, vol. 59, no. 3, pp. 761-769, Mar. 2012.

[21] Y. Katoh, L. L. Snead, I. Szlufarska, and W. J. Weber,
"Radiation effects in SiC for nuclear structural applications," Curr. Opin. Solid State Mater. Sci., vol. 16, no. 3, pp. 143152, Jun. 2012. 